\title{
A Precise Controllable Projection System for Projected Virtual Characters and Its Calibration
}

\author{
Jochen Ehnes* \\ The University of Edinburgh
}

\begin{abstract}
In this paper we describe a system to project virtual characters that shall live with us in the same environment. In order to project the characters' visual representations onto room surfaces we use a controllable projector.
\end{abstract}

\section{INTRODUCTION}

Dialogue systems are applied frequently to automate telephone services. However, it appears to be that at least some people seem to feel slightly uncomfortable to talk to such a system as a voice in the room without a visual point of focus to it.

The expectation to be able to see the dialogue partner can be an important factor if we for example intend to create reminder and support systems for elderly people living at home by themselves, one of our future goals. Those people, who may be suffering from certain forms of dementia, could get very confused if there appears to be a voice talking to them but they cannot connect the voice to a person. Obviously this has to be avoided, or the system might do more damage than it provides support.

With our current project we aim at creating a visual representation of virtual characters that shall live in our environment, created by an extended dialogue system. With this system we plan to verify the existence this effect and investigate how well different types of visual points of focus or virtual characters may help to compensate for it.

\section{Related Work}

The Everywhere Displays project [9] uses a controllable mirror and a steerable camera to project interfaces onto different surfaces. However this system requires a few seconds to switch between different pre-programmed display locations. So while this system is able to create different fixed projection areas, it can not let a virtual character walk across the room in one continuous motion.

The author himself developed an AR system based on controllable video projectors $[2,3]$. This included a roaming architecture that enabled the applications responsible for the augmentation to migrate between different projection units in order to follow the objects and users. User tests were performed to examine the quality of projection as perceived by human subjects dependent on projection distance and angle in order to optimise the selection of the active projection unit at runtime.

In order to be able to augment the environment with a pan- and tilt-able projector, the projector should ideally be placed so that the centre of projection and the pivoting point coincide. Mitsugami et al built such a projection system and described how to move the projector to exactly the right spot in the gimbal [8]. As we use an off the shelf controllable projection system which does not allow for the projector to be moved within the gimbal, we could not use their method of calibration. Yet we were inspired by their use of

*e-mail: jehnes@inf.ed.ac.uk

IEEE International Symposium on Mixed and Augmented Reality 2010 Science and Technolgy Proceedings

13 -16 October, Seoul, Korea

978-1-4244-9345-6/10/\$26.00 @2010 IEEE two projection surfaces at different distances in order to calibrate the system.

For completeness sake we also have to mention efforts in the field of projector camera research on calibration, such as [10, 4]. However, while it is impressive to be able to measure geometrical changes of the projection surfaces with imperceptible structured light or to be able to move an intelligent projection unit, which in turn compensates for that and realigns its projected image with that of its partner units in about ten seconds, it is not really relevant for our application. For once, our controllable projection units are usually mounted in a fixed location. For that reason that location needs to be calibrated only once and even if we move our system once in a while it does not warrant the effort to set up a camera based calibration system, at least not at this stage. On the other hand, when the projector is rotated, the image it projects has to be adapted instantaneously (in fact before the projector even moves to compensate for rendering delays) and a delay of ten seconds would be completely unacceptable.

Kruppa and colleagues $[6,5]$ developed a system to migrate and project virtual characters in a museum environment. Although these virtual characters were able to guide people around an exhibition, they moved around on users' PDAs and only at certain exhibits they could migrate from the PDA to the wall next to the exhibit and back. Users would interact with these characters only through their PDAs. Furthermore, the virtual characters were created using a fixed set of animations, leading to visible inaccuracies when the virtual room inhabitant walked along the wall, projected by a controllable projector [7]. In order to cope with this, the characters were usually transformed into a more abstract form, such as a circle or ball, that could be moved without animation.

\section{Calibration of projector and virtual camera:}

As the projector is mounted in a way that puts is centre of gravity into the pivot point, its lens sits clearly in front of that point. Furthermore the projector is usually not perfectly aligned within the gimbal. Consequently the virtual camera has to be moved forward and rotated slightly from the origin of the pan and tilt device, the pivot point.

As the centre of projection or the point that corresponds to the pinhole in the pinhole camera model is not clearly defined for the projectors lens, we devised a way to optimise this offset parameter together with the view angle, which has to match the projection angle of the lens. As both parameters determine the projection, it was impossible to determine any one of them by projecting onto a single surface only. Instead one can only determine them by adjusting them for two projection surfaces at different distances.

We calibrated these values by projecting a grid pattern onto two surfaces at different distances from the gimbals pivot point and adjusting both values until we got the exact grid size on both surfaces (figure 1). In order to do so, we wrote a set of vertex and fragment shaders that renders any triangle or polyhedron overlaid with a 10 $\mathrm{cm}$ grid based on the vertex coordinates. As the next step we set up two projection surfaces in known distances (the closer one being about half way between the projector and the second one) to the projection system's pivot point and rendered two quads at these 


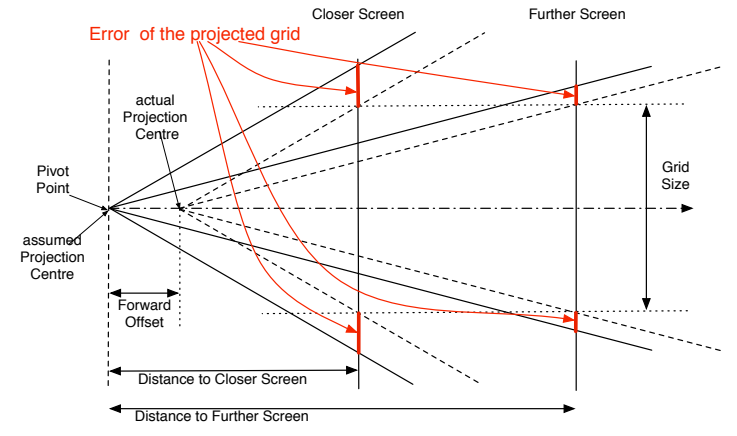

Figure 1: If the distance between the centre of projection and the two projection screens is is wrong it leads to different errors of the projected grid on both screens. This can be used to find the offset of the centre of projection in relation to the gimbal's pivot point, as the distance between the pivot point and the two screens can be measured accurately. As a wrong value of the field of view parameter affects the projections on both screens to the same degree, it can be distinguished from a wrong forward offset and hence both can be adjusted correctly.

distances from the origin. Then we adjusted the field of view as well as the offset of the camera from the origin (i.e. the distance of the projection centre from the pivot point) until the gridlines on both projection surfaces were exactly $10 \mathrm{~cm}$ apart. A good way of doing that is to adjust the field of view to the get the $10 \mathrm{~cm}$ grid on the distant plane. If the grid on the closer surface is smaller than 10 $\mathrm{cm}$, it means that the projector is actually closer than it should be and hence the virtual camera has to be moved closer towards both screens by increasing the offset to compensate for that. If the grid size is bigger than $10 \mathrm{~cm}$, the offset has to be decreased accordingly. While this may sound tedious, as it involves manual tweaking of two parameters, it can easily be done in less that 10 minutes based on the rule described above and illustrated in figure 1. As this has to be done only once for a device, if not only once for all devices of the same make, we did not see a need to try and automate this process. However, if large numbers of devices were to be calibrated, this could be automated by visually comparing the projected grid to calibration patterns on the two screens with two cameras similarly as it has been done by Ashdown et al in [1].

With the offset along the $\mathrm{Z}$ axis known, we have to determine the offset in $\mathrm{X}$ an $\mathrm{Y}$ direction from the pivot point as well as calibrate the alignment of the projector's optical axis with the camera's Zaxis. This can be done by tilting the projector up 90 degrees to align its optical axis with the pan axis. Any misalignment becomes obvious if the projected image does not rotate around the projected image of a point along this axis and can be compensated for. In order to determine an offset, one has to do this for two projection distances as well.

We also developed a method to calibrate the position and orientation of the controllable projection system in the room by aiming it at a few known points, but we don't have the space to describe it in this publication. The same is true for the motion simulation of the controllable projector necessary to register the projection with the environment. [3] describes why that is necessary and illustrates this with a different projection device.

\section{Virtual Characters that 'live' in the environ- MENT}

In contrast to the work by Kruppa et al. [5], we wanted to develop virtual characters that exist continuously in the physical environment, moving across the surfaces of the room in a believable way.
From our perspective the main weakness of their Virtual Room Inhabitant was that it could not move around the room in a believable way. The two main reasons for that as we see it are the use of a set of predefined animations and the use of a human like character.

Our system's design addresses both of these points. For once, the motions of the characters are not predefined, but based on physical simulations of the characters as they move and the generated speech as they talk.

And second, as human-like characters and their way of moving around are not very suitable as projected characters, we decided to use characters in the form of cartoon animals, such as a gecko that can walk on walls as well as on the floor, ceiling or tabletop. This way the characters can walk around obstacles in a believable way. Even very cluttered rooms usually have plenty of space for characters to move around on the ceiling. Furthermore, since such animals can usually only be seen from a certain distance and they have a relatively flat appearance to start with, we expectthat human beings should find their projected images much more acceptable than projected Human like characters.

\section{ACKNOWLEDGEMENTS}

This work is supported by the Marie Curie Intra European Fellowship (IEF) FP7-221125 (NIPUI). This paper only reflects the authors' views and funding agencies are not liable for any use that may be made of the information contained herein.

\section{REFERENCES}

[1] M. Ashdown and Y. Sato. Steerable projector calibration. In Proceedings of IEEE International Workshop on Projector-Camera Systems $2005,2005$.

[2] J. Ehnes and M. Hirose. Projected reality - content delivery right onto objects of daily life. The International Journal of Virtual Reality, 5(3):17-23, September 2006.

[3] J. Ehnes, K. Hirota, and M. Hirose. Projected augmentation - augmented reality using rotatable video projectors. In "ISMAR2004 The Third IEEE and ACM International Symposium on Mixed and Augmented Reality", pages 26-35. IEEE Computer Society, 2004.

[4] T. Johnson, G. Welch, H. Fuchs, E. la Force, and H. Towles. A distributed cooperative framework for continuous multi-projector pose estimation. In VR '09: Proceedings of the 2009 IEEE Virtual Reality Conference, pages 35-42, Washington, DC, USA, 2009. IEEE Computer Society.

[5] M. Kruppa. Migrating Characters: Effective User Guidance in Instrumented Environments. PhD thesis, Saarland Univesity, Saarbrücken, Germany, 2006.

[6] M. Kruppa, A. Krueger, C. Rocchi, O. Stock, and M. Zancanaro. Seamless personalized TV-like presentations on mobile and stationary devices in a museum. In Proceedings of the International Conference on Hypermedia and Interactivity in Museums (ICHIM), Paris, France, 2003.

[7] M. Kruppa, M. Spassova, and M. Schmitz. The virtual room inhabitant - intuitive interaction with intelligent environments. In S. Zhang and R. Jarvis, editors, Proceedings of the 18th Australian Joint Conference on Artificial Intelligence (AI05), Sydney, Australia, 2005.

[8] I. Mitsugami, N. Ukita, and M. Kidode. Multi-planar projection by fixed-center pan-tilt projectors. In CVPR '05: Proceedings of the 2005 IEEE Computer Society Conference on Computer Vision and Pattern Recognition (CVPR'05) - Workshops, page 108, Washington, DC, USA, 2005. IEEE Computer Society.

[9] C. S. Pinhanez. The everywhere displays projector: A device to create ubiquitous graphical interfaces. In UbiComp '01: Proceedings of the $3 r d$ international conference on Ubiquitous Computing, pages 315331, London, UK, 2001. Springer-Verlag.

[10] R. Raskar, G. Welch, M. Cutts, A. Lake, L. Stesin, and H. Fuchs. The office of the future: A unified approach to image-based modeling and spatially immersive displays. Computer Graphics, 32(Annual Conference Series):179-188, 1998. 